\title{
O USO DE METILFENIDATO ENTRE ESTUDANTES UNIVERSITÁRIOS NO BRASIL: UMA REVISÃO SISTEMÁTICA
}

\section{THE USE OF METHYLPHENIDATE AMONG UNIVERSITY STUDENTS IN BRAZIL: A SYSTEMATIC REVIEW}

\author{
Milena de Souza Praxedes', Geovan Figueirêdo de Sá-Filho ${ }^{\| *}$
}

\begin{abstract}
Resumo. O metilfenidato é um medicamento capaz de causar alterações comportamentais, de humor e, devido seus efeitos psicoestimulantes, vem sendo utilizado de forma indiscriminada entre os estudantes para melhorar o desempenho acadêmico. $\mathrm{O}$ estudo teve como objetivo avaliar a prevalência do uso de metilfenidato entre estudantes universitários, bem como, seus efeitos. A pesquisa é uma revisão sistemática de literatura, em que foram reunidos artigos de forma abrangente e não tendenciosa sobre o uso indiscriminado de metilfenidato entre estudantes universitários. Os artigos foram escolhidos a partir das bases eletrônicas SCIELO, Google Acadêmico e LILACS e selecionados 27, publicados entre os anos de 2008 e 2019. O Rio Grande do Sul apresentou a maior quantidade de artigos sobre o tema, entretanto, os estados do Rio de Janeiro e São Paulo manifestaram os maiores números para o uso indiscriminado do medicamento entre os estudantes, nos anos de 2013 e 2014. Registraram-se 17 efeitos negativos em relação ao consumo da droga. São eles: taquicardia/palpitação, boca seca, perda de apetite, ansiedade, cefaleia, insônia, cansaço após uso, náuseas, aumento do estresse, dose dependência, tremores, tremores nas mãos, arritmia, anorexia, declínio da sensação de bemestar, visão turva e euforia. Nesse sentido, foi possível verificar um levantamento geral, do período de 2008 a 2019, sobre a prática do uso inadequado do metilfenidato pelos acadêmicos brasileiros, verificando os benefícios e os malefícios dessa prática inconsequente. Os dados compilados servirão como parâmetro para outras pesquisas, assim como para o desenvolvimento de medidas preventivas e de conscientização.
\end{abstract}

PALAVRAS-CHAVE: Acadêmicos. Melhorador Cognitivo. Off-label. Ritalina.

Abstract. Methylphenidate is a drug capable of causing behavioral and mood changes which, due to its psychostimulating effects, has been used indiscriminately among students to improve academic performance. The study aimed to assess the prevalence of methylphenidate use among university students, as well as its effects. The research is a systematic literature review, in which articles were collected in a comprehensive and non-biased way on the indiscriminate use of methylphenidate among university students. The articles were chosen from the electronic databases SCIELO, Google Scholar and LILACS. The population and sample were the collected articles related to the use of methylphenidate by academics in Brazil, through the inclusion and exclusion criteria. 27 articles were selected between 2008 and 2019. Rio Grande do Sul presented the largest number of articles on the topic; however, the states of Rio de Janeiro and São Paulo showed the greatest results for the indiscriminate use of the medication among students in 2013 and 2014. There were 17 negative effects regarding the use of the drug, which were: tachycardia/palpitation, dry mouth, loss of appetite, anxiety, headache, insomnia, tiredness after use, nausea, increased stress, dose dependence, tremors, hand tremors, arrhythmia, anorexia, decline in sense of well-being, blurred vision and euphoria. In this sense, it was possible to identify a general outcome, between 2008 and 2019, on the inappropriate use of methylphenidate by Brazilian academics, examining the benefits and harms of this careless practice. The compiled data will serve as a parameter for future research, as well as for the development of preventive and awareness measures.

KEYWORDS: Academics. Cognitive Enhancer. Off-label. Ritalin. 


\section{INTRODUÇÃO}

Os medicamentos psicotrópicos têm capacidade de causar alterações comportamentais e de humor. Dentre a ampla gama de medicamentos pertencentes a essa classe, o cloridrato de metilfenidato requer controle especial, de acordo com o disposto na portaria $n^{\circ} 344 / 98$, devido ao alto potencial de causar dependência física ou psíquica. Ele é classificado como um fármaco estimulante do sistema nervoso central (SNC) e atua aumentando a euforia e o estado de vigília. Também conhecido comercialmente por Ritalina ${ }^{\circledR}$, o metilfenidato é aprovado para o tratamento de transtorno de déficit de atenção e hiperatividade (TDAH) e narcolepsia, sendo mais utilizado para o TDAH. ${ }^{1}$

Além de seu efeito terapêutico no TDAH, o uso off-label (termo em inglês para "fora da descrição", ou seja, uso não prescrito) dessa substância vem ganhando espaço na atualidade. Devido seus efeitos psicoestimulantes, o fármaco vem sendo usado para aprimoramento cognitivo em indivíduos saudáveis ${ }^{2}$. Esse tipo de comportamento é observado de forma crescente entre estudantes universitários. A sobrecarga acadêmica, ao ingressar na faculdade, leva os jovens a procurarem a "pílula da inteligência" - como é conhecida entre eles - para desenvolver suas tarefas em um menor espaço de tempo. Por esse motivo, se tornam um grupo de alto risco para o uso indiscriminado dessa substância, justamente pelas cobranças e exigências requeridas nessa fase da vida. 3

Quando um medicamento é aprovado para determinada patologia, ele passa por uma série de estudos que comprovam sua segurança e eficácia para o grupo destinado ao seu uso. Sabendo disso, é difícil medir as consequências do uso do metilfenidato para aprimoramento cognitivo, visto que o medicamento não possui testes clínicos que confirmem sua eficácia em indivíduos saudáveis para essa finalidade. 4

Além da insegurança da eficácia da substância, a falta de orientação em relação ao seu uso pode colocar a saúde do indivíduo em risco. As várias formas de aquisição, como desvios deprescrição, diagnóstico fraudulento e ainda aquisição pela internet, favorecem o uso indiscriminado entre usuários leigos de informações que seriam importantes para o uso correto. As consequências vão desde importantes interações medicamentosas, ingestão de doses tóxicas, agravo de alguma patologia já existente e até mesmo levar o indivíduo a dependência. 5

São vários os estudos que apontam para o crescimento do consumo do metilfenidato. Em vista disso, crescem também as perguntas em relação ao uso indiscriminado dessa substância para fins fora da descrição terapêutica. ${ }^{6}$ Quando o uso é feito para outro fim, que não o do próprio medicamento, fica difícil medir suas consequências, devido à escassez de estudos sobre essa abordagem. Por se tratar de um medicamento de controle especial, segundo a portaria $n^{\circ} 344 / 98$, a vida dos usuários pode estar em risco quando utilizado de forma indiscriminada. 4

O controle especial do metilfenidato, portanto, é fator que deve ser levado em consideração, principalmente se existem dados a respeito do seu uso indiscriminado por algum grupo de indivíduos. Além do grande potencial que o medicamento tem de causar dependência física ou psíquica, também é uma ameaça para a integridade cerebral, por se tratar de um fármaco que causa alterações neurológicas, sendo capaz de desencadear distúrbios emocionais, 
doenças mentais, e ainda, causar importantes alterações sobre os sistemas: cardiovascular, gastrointestinal e endócrino.7 Sabendo que estudantes estão cada vez mais fazendo uso off-label de Ritalina ${ }^{\circledR}$ para melhorar a capacidade cognitiva durante os estudos, se faz necessário considerar os danos em potencial que essa prática possa desencadear.

Dessa forma, se torna indispensável a discussão sobre esse tema uma vez que não se trata apenas de um problema de saúde individual, mas coletiva, e levanta inúmeras questões socioculturais relacionadas às exigências que os estudantes recebem nessa etapa da vida. Exigências que são tanto da família quanto da sociedade, fazendo com que os jovens procurem o medicamento, ultrapassando, assim, os limites do próprio corpo para atender às expectativas. ${ }^{8}$

\section{MATERIAL E MÉTODOS}

A presente pesquisa pode ser considerada uma revisão sistemática de natureza básica, que reuniu informações de forma abrangente e não tendenciosa sobre o uso indiscriminado de MFD entre universitários, gerando um apanhado científico para discussões e apontamentos futuros, reunindo artigos que tratam do uso do metilfenidato por estudantes universitários no Brasil. Os critérios de inclusão foram artigos científicos publicados em periódicos, cuja amostra foi composta por
Além disso, conhecer os dados que tratam dessa temática é fundamental para uma abordagem sobre o impacto aos estudantes, de forma a auxiliar, com informações mais detalhadas sobre o assunto, facilitando a criação de políticas públicas e de prevenção, conscientizando-os para as consequências do uso indiscriminado do metilfenidato.

Tendo em vista o aumento do consumo da substância nos últimos anos, o metilfenidato é o medicamento psicoestimulante sintético mais vendido no mundo, ${ }^{9}$ o objetivo desse estudo é reunir, de forma sistemática, os trabalhos da literatura a respeito do uso do referido remédio, entre estudantes universitários no Brasil, avaliando seus efeitos benéficos e adversos em decorrência do seu consumo. universitários do Brasil, e publicados entre os anos de 2008 e 2020. Os critérios de exclusão foram os artigos que tinham como modelo os indivíduos diagnosticados com TDAH, além de pesquisas realizadas com universitários de outros países.

\section{Instrumento e procedimento de coleta de dados}

Os artigos foram escolhidos mediante pesquisa nas plataformas científicas LILACS, SCIELO e Google Acadêmico (FIGURA 1),

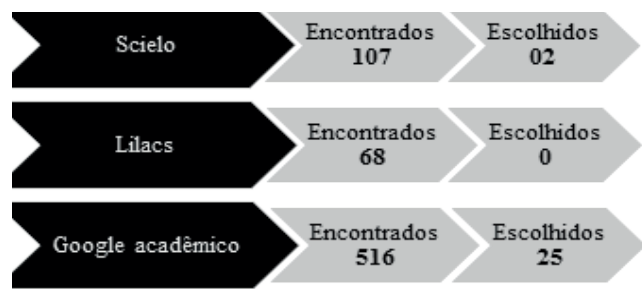

FIGURA 1: Esquema ilustrativo do processo de seleção de artigos encontrados nas bases de dados LILACS, SCIELO e Google Acadêmico, publicados entre os anos de 2008 e 2019, sobre a utilização do metilfenidato por universitários brasileiros 
utilizando os seguintes Descritores em Ciências da Saúde (DeCS - http://decs.bvs.br/): "metilfenidato", "ritalina", "estudantes", "off-label" e "melhorador cognitivo", sendo combinadas com o auxílio dos operadores booleanos "AND" e "OR". Após triagem foram selecionados 27 artigos. Os artigos escolhidos foram analisados por dois avaliadores, pesquisadores doutorandos em ciências farmacêuticas e psicobiologia, respectivamente. Existindo concordância, entre os pesquisadores, na seleção dos artigos de acordo com os critérios de inclusão e exclusão, esses foram utilizados no presente estudo, aumentando a confiabilidade, sendo na sequência avaliados de forma individual e em seguida de forma cruzada.

\section{RESULTADOS E DISCUSSÃO}

Foram encontrados, inicialmente, 764 artigos sobre a utilização do metilfenidato por estudantes universitários brasileiros. Desses, apenas 27 foram utilizados como amostra final, se encaixando nos critérios de inclusão e exclusão estabelecidos. Descartou- se um grande número de pesquisas por não tratarem do uso do medicamento no Brasil. Os efeitos negativos, ou positivos, do uso de metilfenidato por estudantes brasileiros, por estado da federação brasileira, estão apresentados detalhadamente na Tabela 1.

TABELA 1: Artigos agrupados por estados brasileiros e seus principais efeitos negativos e positivos em universitários com relação a utilização do metilfenidato, publicados entre os anos de 2008 e 2019

\begin{tabular}{|c|c|c|c|c|}
\hline Estado & $\begin{array}{l}\text { Números } \\
\text { de artigos }\end{array}$ & Autoria & Efeitos Negativos & Efeitos Positivos \\
\hline Tocantins (TO) & 1 & Silva et al. (2016) & $\begin{array}{c}\text { Taquicardia, palpitação, perda de apetite, } \\
\text { ansiedade, boca seca, tremores, } \\
\text { arritmia, náusea }\end{array}$ & $\begin{array}{l}\text { Aumento do rendimento } \\
\text { acadêmico }\end{array}$ \\
\hline Amazonas (AM) & 1 & luras et al. $(2016)^{11}$ & Não informado & Não informado \\
\hline Pará (PA) & 1 & Monteiro et al. (2019) ${ }^{12}$ & Não informado & Não informado \\
\hline Rondônia (RO) & 5 & $\begin{array}{l}\text { Alberto et al. }(2017)^{13} \\
\text { Junior }(2019)^{14} \\
\text { Menezes et al. }(2020)^{15} \\
\text { Bastos }(2016)^{6} \\
\text { Wille \& Salvi }(2018)^{16}\end{array}$ & $\begin{array}{c}\text { Boca seca, cefaleia, insônia, palpitação, } \\
\text { perda de apetite, irritação, visão turva, } \\
\text { náusea }\end{array}$ & $\begin{array}{l}\text { Aumento do rendimento } \\
\text { acadêmico }\end{array}$ \\
\hline $\begin{array}{l}\text { Minas gerais } \\
\text { (MG) }\end{array}$ & 4 & $\begin{array}{l}\text { Pires et al. }(2018)^{17} \\
\text { Calazans \& Belo }(2016)^{18} \\
\text { Silveira et al. }(2015)^{19} \\
\text { Coli et al. }(2016)^{20}\end{array}$ & $\begin{array}{c}\text { Cansaço após uso, dose dependência, } \\
\text { ansiedade, insônia, euforia, taquicardia, } \\
\text { perda de apetite, aumento do estresse, } \\
\text { cefaleia, tremores }\end{array}$ & $\begin{array}{l}\text { Aumento da concentração } \\
\text { Aumento do rendimento } \\
\text { acadêmico }\end{array}$ \\
\hline $\begin{array}{l}\text { Rio de Janeiro } \\
\text { (RJ) }\end{array}$ & 2 & $\begin{array}{c}\text { Carneiro et al. }(2013)^{2} \\
\text { Mota \& Pessanha }(2014)^{21}\end{array}$ & $\begin{array}{l}\text { Taquicardia, ansiedade, tremores, } \\
\text { anorexia, doca seca, cansaço } \\
\text { após uso, dose dependência }\end{array}$ & $\begin{array}{l}\text { Aumento da concentração } \\
\text { Aumento do rendimento } \\
\text { acadêmico }\end{array}$ \\
\hline $\begin{array}{l}\text { São Paulo } \\
\quad(\mathrm{SP})\end{array}$ & 2 & $\begin{array}{c}\text { Pasquini }(2013)^{22} \\
\text { Bilitardo et al. }(2017)^{23}\end{array}$ & $\begin{array}{c}\text { Taquicardia, perda de apetite, tremores nas } \\
\text { mãos, ansiedade, boca seca, } \\
\text { dose dependência }\end{array}$ & Aumento da concentração \\
\hline Bahia (BA) & 1 & Cruz et al. $(2011)^{24}$ & Não informado & Não informado \\
\hline Paraíba (PB) & 1 & Araújo (2019) 25 & Insônia, ansiedade, taquicardia & $\begin{array}{l}\text { Redução da fadiga e irritabilidade } \\
\text { Aumento da concentração } \\
\text { Melhora no raciocínio } \\
\text { Melhora no bem-estar e memória } \\
\text { Redução do estresse }\end{array}$ \\
\hline
\end{tabular}


CONTINUAÇÃO

\begin{tabular}{|c|c|c|c|c|}
\hline Estado & $\begin{array}{l}\text { Números } \\
\text { de artigos }\end{array}$ & Autoria & Efeitos Negativos & Efeitos Positivos \\
\hline Paraná (PR) & 2 & $\begin{array}{l}\text { Cordeiro \& Pinto }(2017)^{26} \\
\text { Tsuda \& Christoff }(2017)^{27}\end{array}$ & $\begin{array}{l}\text { Aumento do estresse, declínio da } \\
\text { sensação de bem-estar }\end{array}$ & Aumento da capacidade cognitiva \\
\hline $\begin{array}{l}\text { Rio Grande } \\
\text { do Sul (RS) }\end{array}$ & 6 & $\begin{array}{l}\text { Roedel et al. }(2017)^{28} \\
\quad \text { Ferraz }(2018)^{29} \\
\quad \text { Rocha }(2016)^{30} \\
\text { Morgam et al. }(2016)^{31} \\
\text { Silveira et al. (2014)32 } \\
\text { Bassols et al. }(2008)^{33}\end{array}$ & $\begin{array}{c}\text { Cansaço após uso, taquicardia, ansiedade, } \\
\text { cefaleia, perda de apetite, cansaço após } \\
\text { uso, boca seca, tremores nas mãos, } \\
\text { náuseas, dose dependência }\end{array}$ & $\begin{array}{l}\text { Aumento do rendimento } \\
\text { acadêmico } \\
\text { Aumento da concentração }\end{array}$ \\
\hline $\begin{array}{c}\text { Distrito Federal } \\
\text { (DF) } \\
\end{array}$ & 1 & Affonso et al. (2016)34 & Taquicardia, insônia, cefaleia, boca seca & Não informado \\
\hline
\end{tabular}

Dentre as regiões geopolíticas do Brasil, o Sul, Sudeste e o Norte apresentaram maior número de publicações sobre o uso de metilfenidato por estudantes universitários. Já as regiões que apresentaram uma quantidade menor de artigos sobre o tema foram as regiões Nordeste e Centro-Oeste (Figura 2).

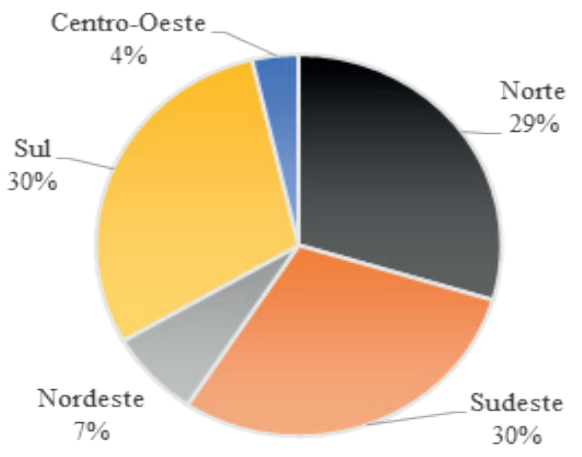

FIGURA 2: Prevalência de publicações científicas sobre a utilização de metilfenidato por universitários nas regiões brasileiras, apresentada em publicações científicas entre os anos de 2008 e 2019

Com relação aos estados brasileiros (Figura 3), o Rio Grande do Sul apresentou maior número de estudos (6 artigos) tratando da temática dentro dos critérios estabelecidos. Em estudos abordando a quantidade de alunos que utilizam o MFD, de forma indiscriminada, foram encontrados valores que variavam de $0 \%$ (0 de 78 alunos entrevistados) a $23 \%$ (34 de 152 entrevistados) dos estudantes. ${ }^{28,29,30,31,32,33}$

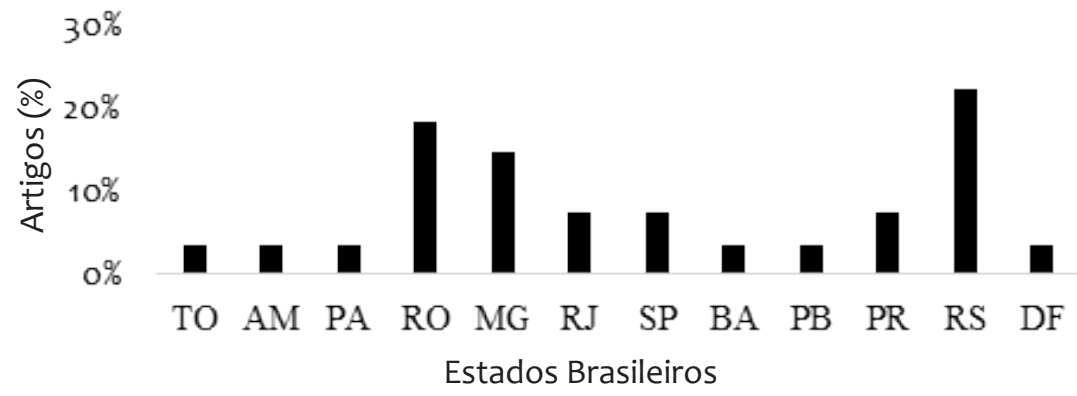

FIGURA 3: Prevalência do uso do metilfenidato por universitários nos estados brasileiros, apresentada em publicações científicas entre os anos de 2008 e 2019 
Logo em seguida Rondônia, com cinco artigos que apresentaram números mais elevados, de 11,11\% (20 de 180 alunos) a 30\% (94 de 315 alunos). 13,14,15,16,23 Em terceiro lugar, Minas Gerais, onde foram localizados quatro artigos, sendo que um deles desenvolveu a pesquisa em duas universidades. Os resultados foram de $5,88 \%$ ( 8 de 136 alunos) a 29,9\% (55 de 187 alunos). ${ }^{17,18,19,20}$

No Paraná26,27, São Paulo 22,23 e Rio de Janeiro ${ }^{2,21}$, foram identificados dois artigos, onde esses dois últimos ( $R J$ e SP) se destacam pelo alto índice de uso indiscriminado do MFD pelos estudantes. Pasquini ${ }^{23}$, aponta, em seu estudo na cidade de São Paulo, que 44,1\% (2286 de 5128 alunos) dos universitários dos mais variados cursos faziam uso indiscriminado do MFD. Mota e Pessanha $^{22}$ realizaram uma pesquisa com alunos de Farmácia e Medicina de uma universidade de Campo de Goytacazes (RJ), onde foi apurado que $60 \%$ (90 de 150 alunos) dos alunos faziam uso indiscriminado da substância.

Em Tocantins ${ }^{10}$, Amazonas ${ }^{11}$, Pará $^{12}$, Bahia ${ }^{24}$, Paraíba ${ }^{25}$ e Distrito Federal (DF), 34 foi encontrado apenas um artigo que abordasse o uso indiscriminado do MFD pelos universitários. No DF34 após uma análise dos dados coletados, de um total de 400 entrevistados, verificou-se que apenas $5,0 \%$ $(n=20)$ relataram o uso de metilfenidato.

Os estados de São Paulo 22,23 e Rio de Janeiro ${ }^{2,21}$ apresentaram os maiores índices de prevalência do uso inadequado do MFD pelos universitários. Constituemse nas metrópoles nacionais onde há maior circulação de capital e desenvolvimento. São regiões que se encontram em um âmbito de desenvolvimento cada vez mais acelerado, com isso, predomina a competitividade em suas várias vertentes, incluindo na área educacional, podendo explicar o alto consumo indiscriminado da substância nesses estados.
O DF, por sua vez, foi onde se encontrou menos consumidor de MFD para fins acadêmicos, todavia, foi identificada apenas uma pesquisa abordando a temática. 34

Nos outros estados como: Acre $(A C)$, Alagoas (AL), Amapá (AP), Ceará (CE), Espírito Santo (ES), Goiás (GO), Maranhão (MA), Mato Grosso (MT), Mato Grosso do Sul (MS), Pará (PA), Pernambuco (PE), Piauí (PI), Rio Grande do Norte (RN), Roraima (RR), Santa Catarina (SC) e Sergipe (SE), não foi encontrado nenhum artigo que tratasse sobre a temática. Os estudos que avaliam o uso de metilfenidato entre estudantes universitários ainda não compreendem os 27 estados brasileiros. Esse fator não permite traçar um perfil concreto dos universitários que utilizam o metilfenidato de forma inadequada, já que estudos por estado são escassos.

Analisado o consumo indiscriminado de MFD pelos estudantes, ao longo do tempo, (entre 2008 e 2019), nos artigos selecionados no presente estudo, percebeu-se que nos anos de 2013 e 2014 houve maior índice no consumo de MFD, respectivamente (Figura 4). Essa informação pode estar relacionada ao aumento dos cursos de graduação presencial nesse mesmo período (2013 e 2014), como informa o INEP (Instituto Nacional de Estudos e Pesquisas), autarquia federal vinculada ao MEC (Ministério da Educação). 35

Isso leva a reflexão de que a grande demanda de atividades e exigências que os estudantes se depararam ao ingressar no ensino superior, os fazem optar por alternativas arriscadas a fim de maximizar seu desempenho. Em relação aos efeitos percebidos pelos estudantes usuários de metilfenidatoemtodosostrabalhosanalisados no presente estudo (Tabela 1), foram registradas algumas reações adversas, sendo relatadas 17 efeitos (Figura 5). A taquicardia/ palpitação são os efeitos mais citados nos artigos (16\%). Na sequência, os efeitos 
boca seca (12\%), perda de apetite ( $9 \%$ ), ansiedade (9\%), cefaleia ( $9 \%$ ), insônia ( $9 \%$ ), cansaço após uso (6\%), náuseas (6\%), aumento do estresse (4\%), dose dependência (ter que aumentar a dose para se obter o mesmo efeito) (4\%), tremores (4\%), tremores nas mãos (3\%), arritmia (1\%), anorexia (1\%), declínio da sensação de bem-estar ( $1 \%$ ), visão turva (1\%) e euforia ( $1 \%$ ).

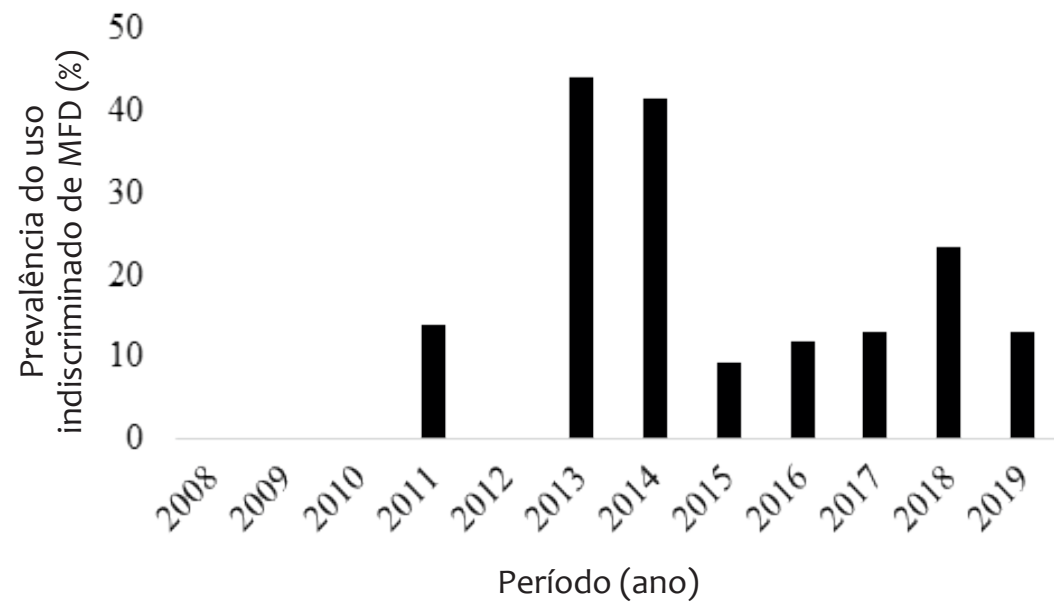

FIGURA 4: Prevalência ddo uso indiscriminado de metilfenidato(MDF) por universitários brasileiros apresentados em publicações científicas entre os anos de 2008 e 2019

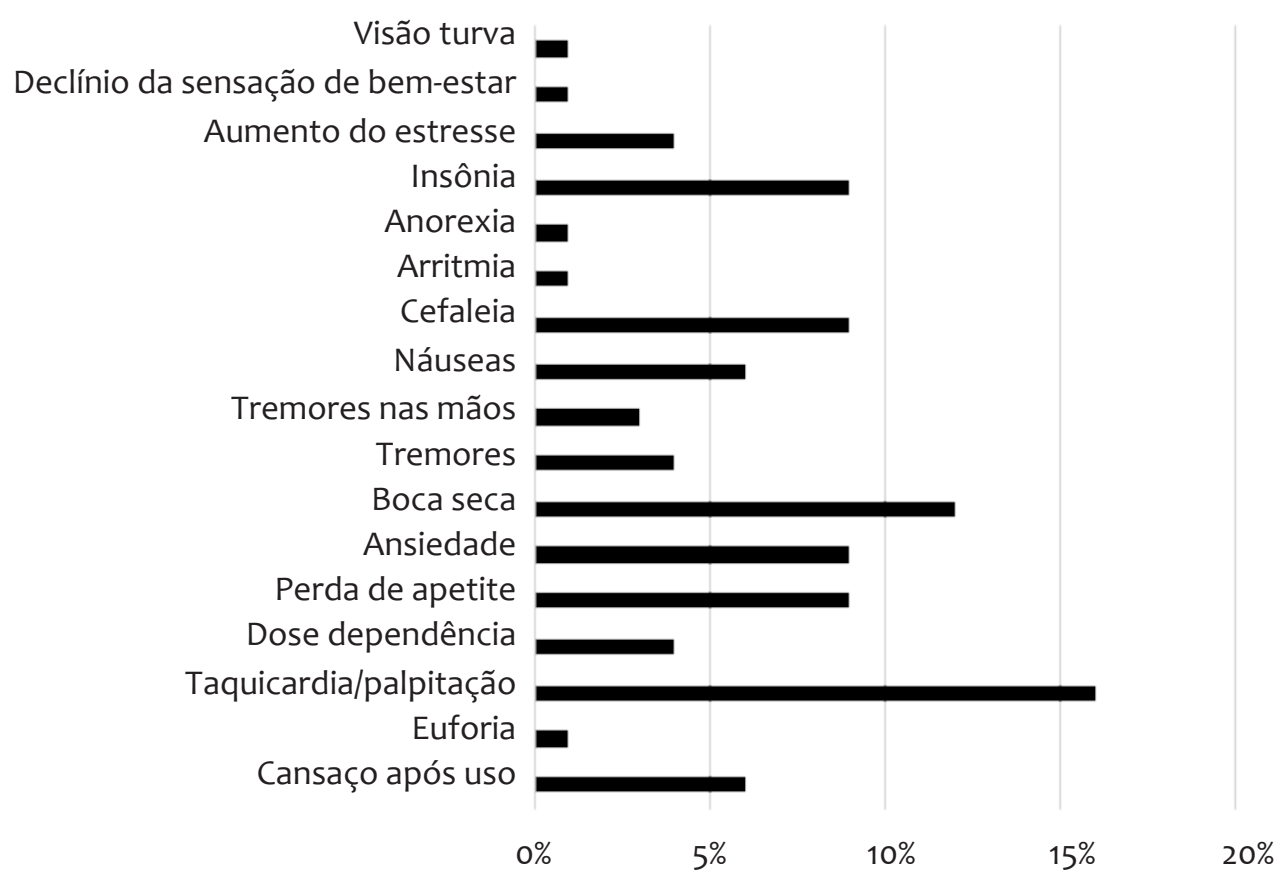

FIGURA 5: Efeitos adversos percebidos por universitários brasileiros, usuários de metilfenidato, apresentados em publicações científicas entre os anos de 2008 e 2019 
Reações adversas, causadas por alterações que o MFD acarretam no sistema cardiovascular, gastrointestinal e nervoso, foram relatadas. Estudo mostra que $60 \%$ dos universitários de todo o Brasil sofrem, ou já sofreram de ansiedade durante a graduação. Outros 32\% de insônia, 30\% já usaram ou usam algum medicamento psiquiátrico, 20\% sofrem de tristeza persistente, $10 \%$ de medo ou pânico, $6 \%$ já apresentaram ideia de morte e $4 \%$ pensamento suicida. ${ }^{36}$ Adicionalmente, o uso de MFD pode agravar esses distúrbios psiquiátricos, fator que ameaça à integridade cerebral dos universitários brasileiros. ${ }^{7}$

Por se tratar de um fármaco capaz de causar alterações neurológicas, tem grande potencial para causar dependência física ou psíquica, e podemos observar esses indícios através do registro "dose dependência" em 4 , das 27 pesquisas $17,23,26,30$, nas quais os estudantes relataram ter que aumentar a dose para obter o mesmo efeito.

Ademais, além da possibilidade de intensificação de problemas psicológicos em usuários de MFD, há também o risco de interações com o medicamento que são, em sua grande maioria, outras substâncias que agem no sistema nervoso central. Como citado acima, 30\% dos acadêmicos de todo Brasil usam, ou já utilizaram psicotrópicos, e o uso concomitante com o MFD acaba sendo nocivo ao usuário, podendo acarretar alterações cardiovasculares, potencialização dos efeitos, sangramentos e outros efeitos adversos, como mostrado na Tabela 1 deste trabalho. 36

Fazendo um compilado, dos 27 artigos avaliados, observam-se alguns efeitos positivos sobre os alunos em relação ao uso da substância (Figura 6), como o aumento da concentração (35\%) e do rendimento acadêmico (40\%), que foram os mais citados. Ainda se destacaram redução da fadiga e irritabilidade (5\%), melhora no raciocínio (5\%), no bem-estar e memória (5\%) e redução do estresse (5\%).

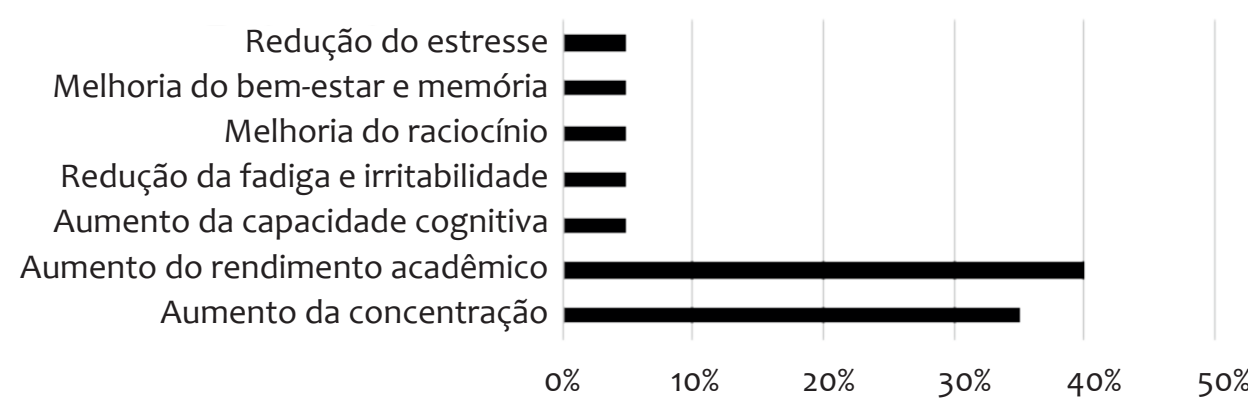

FIGURA 6: Efeitos positivos percebidos por universitários brasileiros, usuários de metilfenidato, apresentados em publicações científicas entre os anos de 2008 e 2019

Grande porcentagem dos estudantes, nos 27 estudos analisados, relataram que o medicamento auxilia no desempenho acadêmico, aumentando a concentração, melhorando o raciocínio, memória, bem-estar e até mesmo reduzindo o estresse. Entretanto, um estudo comprova que o medicamento não possui eficácia em indivíduos saudáveis, ou seja, aqueles que não possuem o déficit, pois não há alterações cerebrais a serem corrigidas. ${ }^{7}$ Contradição, que dá abertura para a possibilidade de futuros estudos de casocontrole, nos quais poderão ser avaliados se o MFD tem ação sobre indivíduos saudáveis, ou se é apenas efeito placebo da droga.

Depois de reparados os efeitos 
relatados pelos universitários, sejam positivos ou negativos, foi considerado que, embora o uso do metilfenidato para auxiliar nos estudos pareça eficaz, o número de efeitos negativos acaba superando essa possível eficácia. As horas aproveitadas de estudos não compensam os malefícios que essa prática

\section{CONSIDERAÇÕES FINAIS}

No presente estudo, os artigos analisados apresentaram pelo menos algum dado que indicasse o uso do metilfenidato entre estudantes acadêmicos. Pôde-se perceber que o consumo indiscriminado é real e que, com frequência, coloca os estudantes em risco, comprometendo sua saúde.

Conclui-se que, mesmo que o uso do metilfenidato apresente efeitos positivos nos universitários no aspecto da aprendizagem cognitiva, é perceptível que os efeitos colaterais podem prejudicar essa positividade. A prática do uso indiscriminado do metilfenidato pode ser porta de entrada para outras doenças e até mesmo causar

\section{REFERÊNCIAS BIBLIOGRÁFICAS}

1. Rang HP, Ritter JM, Flower RJ, Henderson G. Rang \& Dale - Farmacologia. $8^{\text {a }}$ ed. Rio de Janeiro: Elsevier; 2016.

2. Carneiro SG, Prado AST, Araujo ECJ, Moura HC, Strapasson JF, Rabelo NF, et al. O uso não prescrito de metilfenidato entre acadêmicos de Medicina. Cadernos UniFOA. 2013; 8(1): 53-59.

3. Barros D, Ortega F. Metilfenidato e aprimoramento cognitivo farmacológico: representações sociais de universitários. Saude Soc., São Paulo, 2011; 20(2): 350-62. pode desencadear, muitas vezes podendo atingir proporções irreparáveis. Contudo, ainda há de se esperar mais estudos de casocontrole, voltados para essa área, para que se possa desmistificar a efetividade da droga em indivíduos que não possuem o déficit, bem como, seus efeitos a longo prazo.

dependência química, isso é prejudicial à saúde, dos universitários especificamente, e esse conhecimento precisa ser disseminado.

Contudo, ainda há necessidade de melhor compreender os fatores sociais e éticos envolvidos nessa temática. Os estudos que avaliam o uso indiscriminado do metilfenidato para essa prática são insuficientes para que se possa fazer uma análise mais completa a nível nacional, o que representou a limitação do estudo. Com isso, fica em aberto o desenvolvimento de pesquisas sobre o uso de metilfenidato entre estudantes universitários nos estados não compreendidos.

4. Pereira MS, Rau C. A prática off-label e os riscos associados à terapia medicamentosa sem orientação médica. IFAR/PUC. 2013; 1-21.

5. Monteiro BMM, Oliveira KM, Rodrigues LA, Fernandes TF, Silva JBM, Viana NAO, et al. Metilfenidato e melhoramento cognitivo em universitários: um estudo de revisão sistemática. SMAD, Rev. Eletrônica Saúde Mental Álcool Drog. (Ed. port.). 2017; 13(4): 232-42.

6. Bastos EF. Uso indiscriminado do metilfenidato entre os acadêmicos de farmácia da faculdade de educação e meio ambiente. Repositório 
Institucional FAEMA. 2016; 39-49.

7. Andrade LS, Gomes AP, Nunes AB, Rodrigues NS, Lemos O, Rigueiras PO, et al. Ritalina uma droga que ameaça a inteligência. Rev. de Medicina e Saúde de Brasília. 2018. 7(1): 99-12.

8. Brant LC, Carvalho TRF. Metilfenidato: medicamento gadget da contemporaneidade. Interface (Botucatu), Botucatu, 2012; 16(42): 623-36.

9. Junior ATT. Perfil dos universitários da área da saúde quanto ao uso de substâncias psicoativas na cidade de Ariquemes - RO. Biblioteca digital USP, Ribeirão Preto. 2019; 128-37.

10. Silva Júnior DS, Costa KS, da Silva DS. Teles FD, Marcolino MMV, Schneid JL. Prevalência do uso de metilfenidato entre acadêmicos de medicina do centro universitário UNIRG - Tocantins. Rev. Cereus, 2016; 8(3): 172-88.

11. Iuras A, Marques AAF, Garcia LFG, Santiago MB, Santana LKL. Prevalência da automedicação entre estudantes da Universidade do Estado do Amazonas (Brasil). Rev. Portuguesa de Estomatologia, Medicina Dentária e Cirurgia Maxilofacial. 2016; 57(2): 104-11.

12. Monteiro PC, Oliveira CLB, Marques NA, Rego RM, Kins NAA, Caldas CAM. A sobrecarga do curso de Medicina e como os alunos lidam com ela. Braz. J. Hea. Rev. 2019; 2(4): 2998-3010.

13. Alberto M, Valiatti TB, Barcelos IB, Salvi JO. Uso do metilfenidato entre acadêmicos no interior de Rondônia. Rev. da Univ. Vale do Rio Verde. 2017; 15(1): 170-78.

14. Junior ATT. Perfil dos universitários da área da saúde quanto ao uso de substâncias psicoativas na cidade de Ariquemes - RO. Biblioteca digital USP, Ribeirão Preto. 2019; 128-37.

15. Lenzi RV, Nomerg KO, Menezes ASS. O uso de psicoestimulantes por acadêmicos de uma instituição de ensino superior do Estado de
Rondônia. Rev, de Ciências da Saúde 2020; 32(3): 98-06.

16. Wille ARF, Salvi JO. Prevalência do uso de metilfenidato em acadêmicos de um centro universitário em JI-Paraná, Rondônia. BJSCR. 2018;24(3):13-19.

17. Pires MS, Dias AP, Pinto DCL, Gonçalves PG, Segheto W. O uso de substância psicoestimulantes sem prescrição médica por estudantes universitários. Rev. científica FAGOC - saúde. 2018; 3(2): 22-29.

18. Calazans AGC, Belo RFC. Prevalência do uso de metilfenidato por estudantes ingressantes nas universidades do município de Sete Lagoas/ MG. Rev. Brasileira de Ciências da Vida. 2017; 5(1): 26-37.

19. Silveira VI, Oliveira, RJF, Caixeta MR, Andrade BBP, Costa RGL, Santos GB. Uso de psicoestimulantes por acadêmicos de medicina de uma universidade do sul de Minas Gerais. Rev. da Univ. Vale do Rio Verde. 2015; 13(2): 186-92.

20. Coli A, Silva M, Nakasu M. Uso não Prescrito de Metilfenidato entre Estudantes de uma Faculdade de Medicina do Sul de Minas Gerais. Rev. Ciências em Saúde. 2016; 6(1): 121-32.

21. Mota JS, Pessanha FF. Prevalência do uso de metilfenidato por universitários de campos dos Goytacazes. Vértices. 2014; 16(1): 77-86.

22. Pasquini NC. Uso de metilfenidato (MFD) por estudantes universitários com intuito de "turbinar? o cérebro. Revista biologia e farmácia, 2013; 9(2): 107-13.

23. Bilitardo IO, Orrutia VFB, Jesus GM, Sanchez FC, Ortiz BB. Análise do uso de metilfenidato por vestibulandos e graduandos de medicina em uma cidade do estado de São Paulo. Rev. Debates em Psiquiatria. 2017; 6-36.

24. Cruz TCSC, Barreto Júnior, EPS, Gama MLM, Maia LCM, Melo Filho MJX, Manganotti Neto O, 
et al. Uso não-prescrito de metilfenidato entre estudantes de Medicina da Universidade Federal da Bahia. Gazeta Médica da Bahia. 2011; 145(1): 3-6.

25. Araújo JS. Consumo de estimulantes cerebrais por estudantes de farmácia da Universidade Federal da Paraíba: prevalência, motivação e efeitos percebidos. Repositório UFPB. 2019; 44.

26. Cordeiro N, Pinto RMC. Consumo de estimulantes cerebrais em acadêmicos da área da saúde na cidade de Ponta Grossa - PR. Visão Acadêmica, [S.I.], 2017; 18(7): 23-45.

27. Tsuda C, Christoff A. Avaliação do padrão de uso de estimulantes em uma faculdade de Curitiba-PR. Cad. Escola de Saúde. 2017; 20-39.

28. Roedel AM, Margarin FX, Paim RSP. Uso de metilfenidato entre estudantes de psicologia de uma instituição de ensino superior da Serra Gaúcha. V Congresso de Pesquisa e Extensão da FSG III Salão de Extensão. 2017; 76-78.

29. Ferraz L, Piato ALS, Anzolin V, Matter GR, Busato MA. Substâncias psicoativas: o consumo entre acadêmicos de uma universidade do sul do Brasil. Momento: diálogos em educação, 2018; 27(1): 371-86.

30. Rocha B. Avaliação da frequência do uso do Metilfenidato por estudantes de Ensino Superior. Repositório Institucional UNISC. 2016; 63.

31. Morgan HL, Petry AF, Licks PAK, Ballester AO, Teixeira KN, Dumith SC. Consumo de Estimulantes Cerebrais por Estudantes de
Medicina de uma Universidade do Extremo Sul do Brasil: Prevalência, Motivação e Efeitos Percebidos. Rev. Bras. Educ. Médica. 2016; 10209.

32. Silveira RR, Lejderman B, Ferreira PEMS, Rocha GMP. Patterns of non-medical use of methylphenidate among 5th and 6th year students in a medical school in southern Brazil. Trends Psychiatry Psychother, Porto Alegre, 2014; 36(2): 101-06.

33. Bassols AMS, Sordi AO, Eizirik CL, Seeger $G$, Rodrigues GS, Reche $M$. A prevalência de estresse em uma amostra de estudantes do curso de medicina da Universidade Federal do Rio Grande do Sul. Revista HCPA, 2008; 28(1): $153-57$.

34. Affonso RS, Lima KS, Oyama YMO, Deuner MC, Garcia DR, Barboza LL, et al. O uso indiscriminado do cloridrato de metilfenidato como estimulante por estudantes da área da saúde da faculdade Anhanguera de Brasília (FAB). Infarma - Ciências Farmacêuticas, [S.I.], 2016; 28(3): 166-72.

35. Ministério da Educação. Os desafios para acelerar o ritmo e a direção da expansão da educação superior. Censo da educação superior 2018. 2019; 2-76.

36. Oliveira LM, Menezes Filho ACP, Porfiro CA. Uso da Passiflora incarnata L. no tratamento alternativo do transtorno de ansiedade generalizada. Research, Society and Development. 2020; 9(11), e2349119487-e2349119487. 